\title{
Nonholonomic Miechanics and Locomotion: The Snakeboard Example
}

\author{
Jim Ostrowski Andrew Lewis Richard Murray Joel Burdick \\ Department of Mechanical Engineering, California Institute of Technology
}

Mail Code 104-44, Pasadena, CA 91125

\begin{abstract}
Analysis and simulations are performed for a simplified model of a commercially available variant of the skateboard, known as the Snakeboard ${ }^{1}$. Although the model exhibits basic gait patterns seen in a large number of locomotion problems, the analysis tools currently available do not apply to this prob. lem. The difficulty lies primarily in the way in which the nonholonomic constraints enter into the system. As a first step towards understanding systems represented by our model we present the equations of motion and perform some controllability analysis for the snakeboard. We also perform numerical simulations of possible gait patterns which are characteristic of snakeboard locomotion.
\end{abstract}

\section{Introduction}

This paper investigates a simplified model of a commercially available derivative of a skateboard known as the Snakeboard. The Snakeboard (Figure 1) allows the rider to propel $\mathrm{him} /$ herself forward without having to make contact with the ground. This motion is roughly accomplished by coupling a conservation of angular momentum effect with the nonholonomic constraints defined by the condition that the wheels roll without slipping. Snakeboard propulsion is discussed in more detail in Section 1.

We study this model for several reasons. First, the snakeboard's means of locomotion has not appeared in prior studies of robotic locomotion. Numerous investigators have studied and successfully demonstrated quasi-static multi-legged locomotion devices [16]. Others have considered and implemented various forms of undulatory, or "snake-like," locomotion schemes [6], [4]. Beginning with Raibert [13], hopping robots have received considerable attention as well $[8,10,1]$. Bipedal walking and running has also been an active area of research $[9,7]$. In all of these cases except [1], the robotic locomotion devices are largely anthropomorphic or zoomorphic. The method of locomotion used for the snakeboard is significantly different from all of these approaches and does not appear to have a direct biological counterpart. There is, however, some similarity to the undulatory motion of snakes, and it is hoped that this research will provide insight into other areas of locomotion which make use of constraints arising through ground contact.

Despite its unique features, the mechanics of the snakeboard's movement has several properties which we believe to be common to many forms of locomotion. In Section 4, the simplified snakeboard model is shown to exhibit a number of gaits, each of which generates a net motion in a certain direction by performing loops in the controlled variables. This general method of locomotion (i.e., generating net motions by cycling certain control variables) appears to be generic

\footnotetext{
${ }^{1}$ The name Snakeboard has been trademarked.
}

to most methods of locomotion, including walking, running, parallel parking, undulating, and sidewinding.

Superficially, the snakeboard appears to be closely related to other robotic systems with nonholonomic constraints, where cyclic motions in the control space of the vehicle can cause net motion in the constrained directions (see [11] for an introduction and references). However, the dynamics of our model of the Snakeboard do not fit into the principal fibre bundle structure which has been used to study some nonholonomic systems [2]. The snakeboard seems to represent a class of systems for which current analysis tools do not provide any assistance. Thus, the snakeboard model: (1) is an interesting problem in nonholonomic mechanics; (2) represents an unexplored class of systems which may be used for locomotion; and (3) serves as a motivating example for the development of new frameworks for exploring the relationship between nonholonomic mechanics and locomotion.

In Section 1 we give a detailed description of the Snake board and how it is used. We also present our simplified model which is intended to capture the essential features of the Snakeboard. In Section 2 we present Lagrange's equations for the snakeboard and describe a control law which allows us to follow specified inputs exactly. Since the snakeboard is modeled as a constrained control system, it is possible to examine controllability and thereby determine whether we may reach all points in our state space. This analysis is presented in Section 3. In Section 4 the above mentioned gaits are presented and analysed. The failure of the snakeboard to fit into a principal bundle formulation is discussed in Section 5. Included in this discussion is the introduction of possible tools suggested by recent research which may make the problem more tractable.

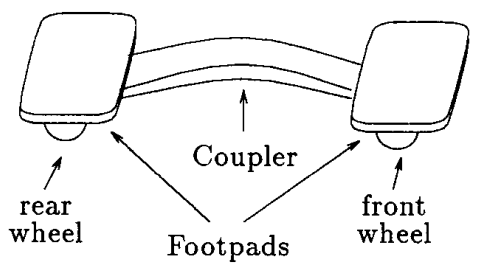

Figure 1: The Snakeboard

\section{The Snakeboard and a simplified model}

The Snakeboard consists of two wheel-based platforms upon which the rider is to place each of his feet. These platforms are connected by a rigid coupler with hinges at each platform to allow rotation about the vertical axis. See Figure 1. To propel the snakeboard, the rider first turns both of his feet 


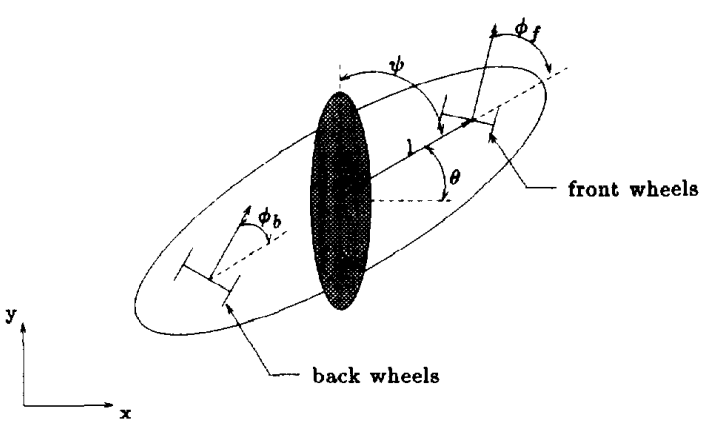

Figure 2: The simplified model of the Snakeboard

in. By moving his torso through an angle, the Snakeboard moves through an arc defined by the wheel angles. The rider then turns both feet so that they point out, and moves his torso in the opposite direction. By continuing this process the Snakeboard may be propelled in the forward direction without the rider having to touch the ground.

Our simplified model of the Snakeboard is shown in Figure 2. We will use the term snakeboard to refer to this model, but will distinguish the model from the commercially available Snakeboard by using italics and capitals to describe the latter. As a mechanical system the snakeboard has a configuration space given by $Q=S E(2) \times S^{1} \times S^{1} \times S^{1}$. Here $S E(2)$ is the group of rigid motions in the plane, and we are thinking of this as describing the position of the board itself. By $\$^{1}$ we mean the group of rotations on $\mathbf{R}^{2}$. The three copies of $\boldsymbol{S}^{1}$ in $Q$ describe the positions of the rotor and the two wheels, respectively. As coordinates for $Q$ we shall use $\left(x, y, \theta, \psi, \phi_{b}, \phi_{f}\right)$ where $(x, y, \theta)$ describes the position of the board with respect to a reference frame (and so are to be thought of as an element of $S E(2)), \psi$ is the angle of the rotor with respect to the board, and $\phi_{b}$, and $\phi_{f}$ are, respectively, the angles of the back and front wheels with respect to the board. We will frequently refer to the variables $\left(\psi, \phi_{b}, \phi_{f}\right)$ as the controlled variables since they are the variables which are rider inputs in the actual Snakeboard. Parameters for the problem are:

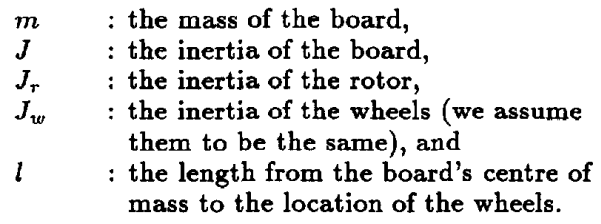

The wheels of the snakeboard are assumed to roll without lateral sliding. This condition is modeled by constraints which may be shown to be nonholonomic. At the back wheels the constraint assumes the form

$$
-\sin \left(\phi_{b}+\theta\right) \dot{x}+\cos \left(\phi_{b}+\theta\right) \dot{y}-l \cos \left(\phi_{b}\right) \dot{\theta}=0 .
$$

Similarly at the front wheels the constraint appears as

$$
-\sin \left(\phi_{f}+\theta\right) \dot{x}+\cos \left(\phi_{f}+\theta\right) \dot{y}+l \cos \left(\phi_{f}\right) \dot{\theta}=0 .
$$

Alternatively one can write the constraints as the kernel of two differential one-forms. To be specific, all velocities must lie in $\operatorname{ker}\left\{\omega^{1}, \omega^{2}\right\}$, where

$$
\begin{aligned}
& \omega^{1}=-\sin \left(\phi_{b}+\theta\right) d x+\cos \left(\phi_{b}+\theta\right) d y-l \cos \left(\phi_{b}\right) d \theta \\
& \omega^{2}=-\sin \left(\phi_{f}+\theta\right) d x+\cos \left(\phi_{f}+\theta\right) d y+l \cos \left(\phi_{f}\right) d \theta
\end{aligned}
$$

\section{Dynamics and control of the snakeboard}

To investigate the dynamics of the snakeboard we use Lagrange's equations which, for constrained and forced systems, are given by

$$
\frac{d}{d t}\left(\frac{\partial L}{\partial \dot{q}^{i}}\right)-\frac{\partial L}{\partial q^{i}}=\sum_{j=1}^{m} \lambda_{j} \omega_{i}^{j}+\tau_{i}, \quad i=1, \ldots, n .
$$

Here $\lambda_{1}, \ldots, \lambda_{m}$ are the Lagrange multipliers, $\omega^{1}, \ldots, \omega^{m}$ are the constraint one-forms, and $\tau_{1}, \ldots, \tau_{n}$ are the external forces. The first term on the right hand side of Lagrange's equations may be regarded as an external force applied to the system to ensure that the constraints are satisfied. As such, the Lagrange multipliers are a part of the solution to the problem. See [12] for a discussion of Lagrangian mechanics in this vein. We will only consider torques on the controlled variables $\psi, \phi_{b}$, and $\phi_{f}$. The Lagrangian for the snakeboard is

$$
\begin{aligned}
L=\frac{1}{2} m\left(\dot{x}^{2}+\dot{y}^{2}\right)+\frac{1}{2} J \dot{\theta}^{2}+ & \frac{1}{2} J_{r}(\dot{\psi}+\dot{\theta})^{2}+ \\
& \frac{1}{2} J_{w}\left(\left(\dot{\phi}_{b}+\dot{\theta}\right)^{2}+\left(\dot{\phi}_{f}+\dot{\theta}\right)^{2}\right) .
\end{aligned}
$$

Thus Lagrange's equations are given by

$$
\begin{aligned}
& m \ddot{x}-\lambda_{1} \sin \left(\phi_{b}+\theta\right)-\lambda_{2} \sin \left(\phi_{f}+\theta\right)=0 \\
& m \ddot{y}+\lambda_{1} \cos \left(\phi_{b}+\theta\right)+\lambda_{2} \cos \left(\phi_{f}+\theta\right)=0 \\
& \left(J+J_{\tau}+2 J_{w}\right) \ddot{\theta}+J_{r} \ddot{\psi}+J_{w} \ddot{\phi}_{b}+J_{w} \ddot{\phi}_{f}- \\
& \lambda_{1} l \cos \phi_{b}+\lambda_{2} l \cos \phi_{f}=0 \\
& J_{r} \ddot{\psi}+J_{r} \ddot{\theta}=u_{1} \\
& J_{w} \ddot{\phi_{b}}+J_{w} \ddot{\theta}=u_{2} \\
& J_{w} \ddot{\phi_{f}}+J_{w} \ddot{\theta}=u_{3}
\end{aligned}
$$

where $\left(u_{1}, u_{2}, u_{3}\right)$ are the input torques in the $\left(\psi, \phi_{b}, \phi_{f}\right)$ directions, respectively.

Since the rider of the Snakeboard typically propels himself by performing cyclic motions with his feet and torso, it was deemed desirable to devise a control law which would allow one to follow any curve, $t \mapsto\left(\psi(t), \phi_{b}(t), \phi_{f}(t)\right)$, in the controlled variables. It turns out that such a control law is derivable with some manipulation of Lagrange's equations. We outline some of this manipulation in the proof of the following proposition.

Proposition 2.1 Let $t \mapsto\left(\psi(t), \phi_{b}(t), \phi_{f}(t)\right)$ be a piecewise smooth curve. Then there exists a control law $t \mapsto$ $\left(u_{1}(t), u_{2}(t), u_{s}(t)\right)$ so that the $\left(\psi, \phi_{b}, \phi_{f}\right)$ components of the solution to Lagrange's equations are given by $t \mapsto$ $\left(\psi(t), \phi_{b}(t), \phi_{f}(t)\right)$.

Proof: In Lagrange's equations, (2.1)-(2.6), and in the constraints, (1.1) and (1.2), regard $\left(\psi, \phi_{b}, \phi_{f}\right)$ as known functions of $t$. Substituting (2.4)-(2.6) into (2.3) gives

$$
\ddot{\theta}=\frac{1}{J}\left(-u_{1}-u_{2}-u_{3}+\lambda_{1} l \cos \phi_{b}-\lambda_{2} l \cos \phi_{f}\right) .
$$


Substituting this expression back into (2.4)-(2.6) gives an expression of the form

$$
B u=P(t)+N(t) \lambda
$$

where $u=\left(u_{1}, u_{2}, u_{3}\right), \lambda=\left(\lambda_{1}, \lambda_{2}\right), B \in \mathbb{R}^{3 \times 3}$ is a known constant matrix, $P(t) \in \mathbf{R}^{3}$ is a known time-dependent vector, and $N(t) \in \mathbf{R}^{3 \times 2}$ is a known time-dependent matrix. Now observe that we may write $(2.1),(2.2)$, and $(2.7)$ as

$$
M \ddot{z}+W^{T}(z, t) \lambda=f .
$$

Here $z=(x, y, \theta), f=\left(0,0,-u_{1}-u_{2}-u_{3}\right), M \in \mathbb{R}^{3 \times 3}$ is a known constant invertible matrix, and $W(z, t) \in \mathbb{R}^{2 \times 3}$ is a known time-dependent matrix function of $z$. The constraints appear as

$$
W(z, t) \dot{z}=0 .
$$

Using (2.9) and (2.10) we may derive

$$
\lambda=\left(W M^{-1} W^{T}\right)^{-1}\left(W M^{-1} f+\dot{W} \dot{z}\right) .
$$

Since $\left(u_{1}, u_{2}, u_{3}\right)$ appear linearly in $f$, we can replace $W M^{-1} f$ with an equivalent representation which is linear in the control torques, $u$ :

$$
W M^{-1} f=C u,
$$

where $C(z, t) \in \mathbb{R}^{2 \times 3}$. Finally, using (2.8), (2.11) and (2.12) we arrive at the formula

$$
\begin{aligned}
u(z, \dot{z}, t)=\left(B-N\left(W M^{-1} W^{T}\right)^{-1} C\right)^{-1} & \\
& \left(P+N\left(W M^{-1} W^{T}\right)^{-1} \dot{W} \dot{z}\right)
\end{aligned}
$$

for the feedback control law which follows a specified trajectory in $\left(\psi, \phi_{b}, \phi_{f}\right)$. The matrices that need to be inverted may be shown to be invertible except at isolated configurations.

\section{Controllability of the snakeboard}

As a control system, one would like to show that the snakeboard is controllable in the following sense: Given two configurations $q_{1}, q_{2} \in S E(2)$ of the board, there exists an input $t \mapsto\left(u_{1}(t), u_{2}(t), u_{3}(t)\right)$ which steers the system from rest at $q_{1}$ to being at rest at $q_{2}$. To prove that the snakeboard is so controllable we first reduce the system from a problem in mechanics to a problem in kinematics. In doing so we reduce the problem to one of finding paths in the configuration variables which lie in a certain distribution. The answer to this problem is then given by Chow's theorem which states that a path lying in the distribution may be found which connects two points if the distribution is maximally involutive (see [5]).

From (2.13) we have a control law which allows us to follow any path in the controlled variables, $\left(\psi, \phi_{b}, \phi_{f}\right)$, we desire. It turns out that this is enough to allow us to follow any path in the variables $\left(x, y, \theta, \phi_{b}, \phi_{f}\right)$ which satisfy the constraints.

Proposition 3.1 Let $c: t \mapsto\left(x(t), y(t), \theta(t), \phi_{b}(t), \phi_{f}(t)\right)$. be a piecewise smooth curve so that $\phi_{b}(t) \neq \phi_{f}(t)$ and so that $c^{\prime}(t) \in \operatorname{ker}\left\{\omega^{1}, \omega^{2}\right\}$ for all $t$. Then there exists a control law $t \mapsto\left(u_{1}(t), u_{2}(t), u_{3}(t)\right)$ so that the $\left(x, y, \theta, \phi_{b}, \phi_{f}\right)$ compo. nents of the solution to Lagrange's equations are given by $t \mapsto\left(x(t), y(t), \theta(t), \phi_{b}(t), \phi_{f}(t)\right)$.
Proof: We shall use notation similar to that in the proof of Proposition 2.1 although the objects will be different. First let $\left(x, y, \theta, \phi_{b}, \phi_{f}\right)$ be regarded as known functions of $t$ in Lagrange's equations and be such that they satisfy the constraints. This immediately specifies $u_{2}(t)$ and $u_{3}(t)$ from $(2.5)$ and (2.6), respectively. If we denote $s=(x, y)$ we may write $(2.1)$ and $(2.2)$ as

$$
M \ddot{s}+W^{T}(t) \lambda=0
$$

where $M \in \mathbb{R}^{2 \times 2}$ is a known constant invertible matrix, $W(t) \in \mathbb{R}^{2 \times 2}$ is the time-dependent matrix given by

$$
W(t)=\left[\begin{array}{cc}
-\sin \left(\phi_{b}(t)+\theta(t)\right) & -\sin \left(\phi_{f}(t)+\theta(t)\right) \\
\cos \left(\phi_{b}(t)+\theta(t)\right) & \cos \left(\phi_{f}(t)+\theta(t)\right)
\end{array}\right],
$$

and $\lambda=\left(\lambda_{1}, \lambda_{2}\right)$. The constraint equations may be written as

$$
W(t) \dot{s}=R(t)
$$

where $R(t) \in \mathbb{R}^{2}$ is a known time-dependent vector. Equations (3.1) and (3.2) may be combined to obtain

$$
\lambda=\left(W M^{-1} W^{T}\right)^{-1}(\dot{W} \dot{s}-\dot{R}(t))
$$

From (2.7) we have a relation of the form

$$
u_{1}=P(t)+N(t) \lambda
$$

where $P(t)$ is a known function of $t$, and $N(t) \in \mathbb{R}^{1 \times 2}$ is a known time-dependent matrix. Now we use (3.3) and (3.4) to get

$$
u_{1}(t)=P(t)+N(t)\left(W M^{-1} W^{T}\right)^{-1}(\dot{W} \dot{s}-\dot{R}(t)) .
$$

Combining this with $u_{2}(t)$ and $u_{3}(t)$ as determined above, we obtain the proposition.

Note the matrix $W(t)$ is invertible if and only if $\phi_{b}(t) \neq$ $\phi_{f}(t)$.

As the proof illustrates, the configurations where $\phi_{b}=\phi_{f}$ cause problems because $W$ is singular at such configurations. Because of these difficulties, one can only track trajectories which go through configurations where $\phi_{b}=\phi_{f}$ and $\dot{\theta}=0$.

Proposition 3.1 now allows us to consider controllability of the nonholonomic system

$$
\dot{q}=u_{1} g_{1}(q)+u_{2} g_{2}(q)+u_{3} g_{3}(q)
$$

where $q=\left(x, y, \theta, \phi_{b}, \phi_{f}\right)$, and $\left\{g_{1}, g_{2}, g_{3}\right\}$ forms a basis for the distribution defined by $\operatorname{ker}\left\{\omega^{1}, \omega^{2}\right\}$ (see (1.3) and (1.4)). As a basis we use

$$
\begin{aligned}
g_{1}= & (0,0,0,1,0) \\
g_{2}= & (0,0,0,0,1) \\
g_{3}= & \left(-l\left(\cos \phi_{b} \cos \left(\phi_{f}+\theta\right)+\cos \phi_{f} \cos \left(\phi_{b}+\theta\right)\right),\right. \\
& -l\left(\cos \phi_{b} \sin \left(\phi_{f}+\theta\right)+\cos \phi_{f} \sin \left(\phi_{b}+\theta\right)\right), \\
& \left.\sin \left(\phi_{b}-\phi_{f}\right), 0,0\right)
\end{aligned}
$$

These vector fields may be shown to span a distribution of rank 3 except when $\phi_{b}=\phi_{f}= \pm \pi / 2$ where the rank drops by 1 . The drop in rank at these points is reflected by there being two directions of admissible motion for the board at these configurations. A simple computation gives

$$
\begin{aligned}
{\left[g_{1}, g_{3}\right]=} & \left(l\left(\cos \left(\phi_{f}+\theta\right) \sin \phi_{b}+\cos \phi_{f} \sin \left(\phi_{b}+\theta\right)\right),\right. \\
& -l\left(\cos \phi_{f} \cos \left(\phi_{b}+\theta\right)-\sin \phi_{b} \cos \left(\phi_{f}+\theta\right)\right), \\
& \left.\cos \left(\phi_{b}-\phi_{f}\right), 0,0\right) \\
{\left[g_{2}, g_{3}\right]=} & \left(l\left(\cos \left(\phi_{b}+\theta\right) \sin \phi_{f}+\cos \phi_{b} \sin \left(\phi_{f}+\theta\right)\right),\right. \\
& -l\left(\cos \phi_{b} \cos \left(\phi_{f}+\theta\right)-\sin \phi_{f} \cos \left(\phi_{b}+\theta\right)\right), \\
& \left.-\cos \left(\phi_{b}-\phi_{f}\right), 0,0\right)
\end{aligned}
$$


The vector fields $\left\{g_{1}, g_{2}, g_{3},\left[g_{1}, g_{3}\right],\left[g_{2}, g_{3}\right]\right\}$ may be shown to span $T_{q} Q$ except at isolated points. Thus, excluding this set of points, the control system given by (3.5) is controllable by Chow's theorem.

\section{Gaits for the snakeboard}

By performing various types of loops in our controlled variables, we found that it was possible to generate gaits for the snakeboard. We will loosely call a gait a periodic motion in the controlled variables which results in a net displacement of the snakeboard.

The use of periodic motions in some variables for trajectory generation has been well studied for nonholonomic systems. For example, Tilbury, Murray, and Sastry, [17], use sinusoids to generate motion in a system with $n$ trailers. Although our system is not directly analogous to the class of systems for which sinusoids have proven to be useful, observations of actual Snakeboard riders suggest that sinusoidal inputs provide a good starting point for our investigations. We consider paths in the controlled variables of the form

$$
\begin{aligned}
& t \mapsto\left(a_{\psi} \sin \left(\omega_{\psi} t+\beta_{\psi}\right), a_{b} \sin \left(\omega_{b} t+\beta_{b}\right),\right. \\
&\left.a_{f} \sin \left(\omega_{f} t+\beta_{f}\right)\right) .
\end{aligned}
$$

A gait will be referenced by a triple $\left(i_{1}, i_{2}, i_{3}\right)$ of integers where $i_{1}=\omega_{\psi}, i_{2}=\omega_{b}$, and $i_{3}=\omega_{f}$. All of the gaits we discuss will have $i_{2}=i_{3}, \beta_{b}=\beta_{f}$, and $a_{b}=-a_{f}$. This corresponds to typical gaits in the Snakeboard where the rider moves his feet in opposing motions.

In the gait simulations below, the following parameters were used: $m=6 \mathrm{~kg}, J=0.016 \mathrm{~kg} \cdot \mathrm{m}^{2}, J_{r}=0.072 \mathrm{~kg} \cdot \mathrm{m}^{2}$, $J_{u}=0.0013 \mathrm{~kg} \cdot \mathrm{m}^{2}$, and $l=0.2 \mathrm{~m}$. These values reflect possible choices which would make physical sense for a working model of the Snakeboard. In discussing these gaits, the snakeboard is assumed to have its initial condition at the origin in the state space. Thus "forward" motion is in the $x$-direction, and "transverse" motion is in the $y$-direction.

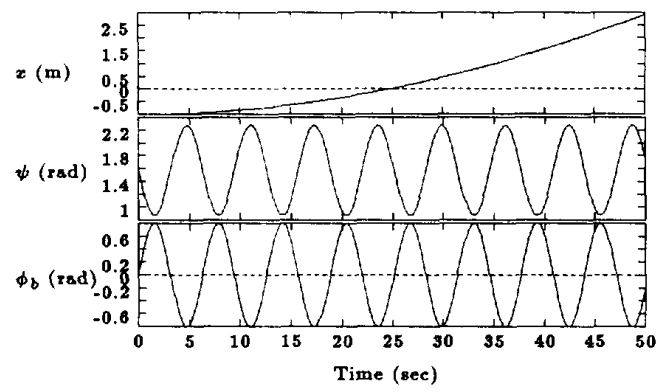

Figure 3: Time histories of the controls and $x$ for the $(1,1,1)$ gait

The "drive" gait: $(1,1,1)$

The drive gait is used to move the snakeboard in the $x$ direction and is determined by the frequencies $(1,1,1)$. Figure 3 shows plots of the controlled variables and $x$, and Figure 4 shows the position of the centre of mass of the snakeboard along the trajectory for the $(1,1,1)$ gait.

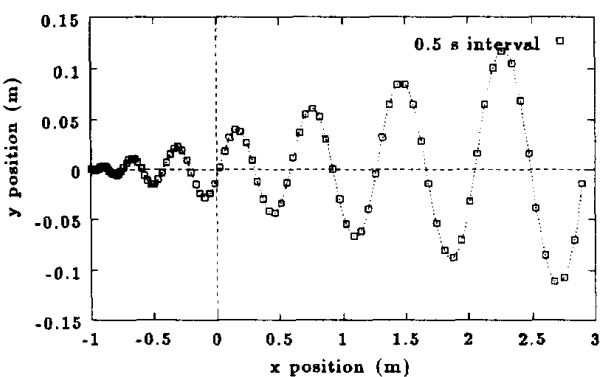

Figure 4: Position of the centre of mass for the $(1,1,1)$ gait

The following parameters were fixed at the indicated values for the analysis of the $(1,1,1)$ gait.

$$
\begin{aligned}
& a_{b},-a_{f}: 0.3 \mathrm{rad} \\
& \omega_{b}, \omega_{f}: 1 \mathrm{rad} / \mathrm{sec} \\
& \beta_{b}, \beta_{f}: 0 \mathrm{rad} \\
& a_{\psi}: 0.7 \mathrm{rad} \text { (unless otherwise specified) } \\
& \omega_{\psi}: 1 \mathrm{rad} / \mathrm{sec} \text { (unless otherwise specified) } \\
& \beta_{\psi}: 0 \mathrm{rad} \text { (unless otherwise specified) }
\end{aligned}
$$

If one fixes the rotor phase angle $\beta_{\psi}$ at 0 the snakeboard will be propelled in the negative $x$-direction. Changing this phase angle to $\pi$ will result in motion in the positive $x$-direction. Figure 5 shows the result of varying $\beta_{\psi}$ from $-\pi / 2$ to $\pi / 2$. Notice that if the rotor moves $90^{\circ}$ out of phase with the wheels, then almost no motion is observed in the $x$-direction.

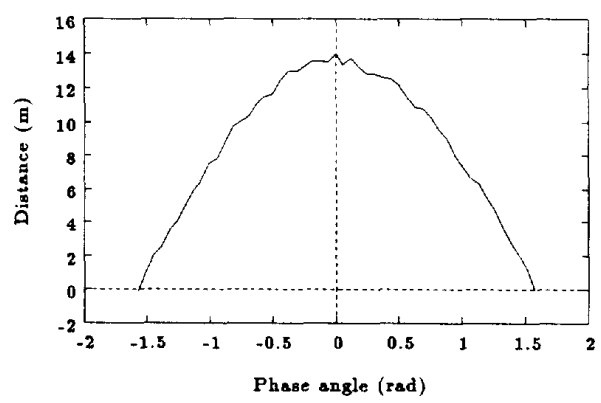

Figure 5: Distance traveled in the (negative) $x$-direction vs $\beta_{\psi}$ for the $(1,1,1)$ gait

Frequency ratios around $(1,1,1)$ were also investigated, but, for generating net motion in the $x$-direction, the $(1,1,1)$ gait was determined to be superior. In Figure 6 we see the relationship between distance traveled in the $x$-direction versus $\omega_{\psi}$ near the operating point $\omega_{\psi}=1$. Four different simulation times, $T$, are shown. A similar analysis was also performed for a wider range of frequencies which demonstrated that the $(1,1,1)$ gait generated more motion in the $x$-direction than any other frequency ratios.

The final parameter study was done on $a_{\psi}$, the amplitude of the rotor swing. The results are shown in Figure 7 . Notice that for large amplitudes and long simulation times the distance traveled begins to decrease. This is a result of the ossillations in the $y$-direction becoming large enough to cause 


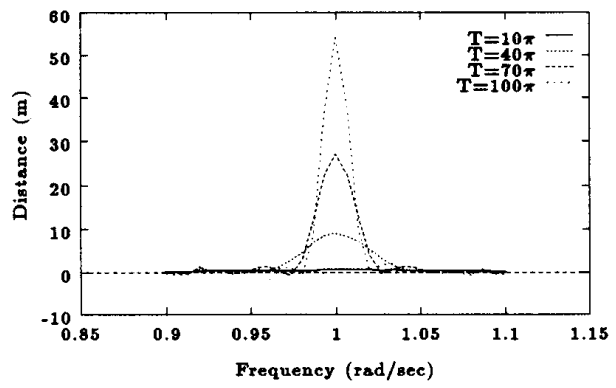

Figure 6: Distance traveled in the $x$-direction vs $\omega_{\psi}$ for the $(1,1,1)$ gait

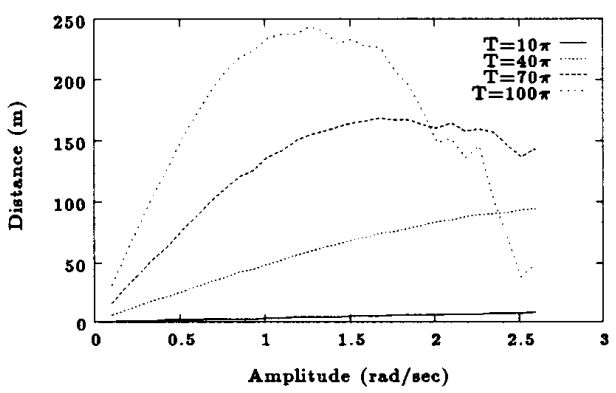

Figure 7: Distance traveled in the $x$-direction vs $a_{\psi}$ for the $(1,1,1)$ gait

the board to actually turn around. Thus one cannot just increase the run time and input magnitude to get longer distances traveled in the $(1,1,1)$ gait.

The "rotate" gait: $(2,1,1)$

The rotate gait generates net motion in the $\theta$-direction by using frequencies $(2,1,1)$. A trace of the centre of mass of the board is shown in Figure 8.

The following parameters were fixed at the indicated values for the analysis of the $(2,1,1)$ gait.

$$
\begin{aligned}
& a_{b},-a_{f}: 1 \mathrm{rad} \\
& \omega_{b}, \omega_{f}: 1 \mathrm{rad} / \mathrm{sec} \\
& \beta_{b}, \beta_{f}: 0 \mathrm{rad} \\
& a_{\psi}: 1 \mathrm{rad} \\
& \omega_{\psi}: 2 \mathrm{rad} / \mathrm{sec} \text { (unless otherwise specified) } \\
& \beta_{\psi}: 0 \mathrm{rad}
\end{aligned}
$$

The relationship between the net angle of rotation and the phase angle, $\beta_{\psi}$, is much the same as was observed in the $(1,1,1)$ gait. That is to say, if the rotor and wheels are out of phase, no net motion is produced in the $\theta$-direction. The result of varying the frequency ratio around the operating point of $(2,1,1)$ is shown in Figure 9. If one increases the amplitude, $a_{\psi}$, for the $(2,1,1)$ gait, the resulting net displacement in $\theta$ will always increase, unlike the situation in the $(1,1,1)$ gait.

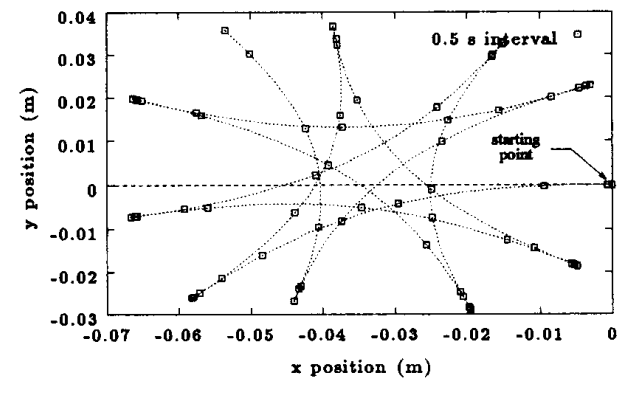

Figure 8: Position of the centre of mass for the $(2,1,1)$ gait

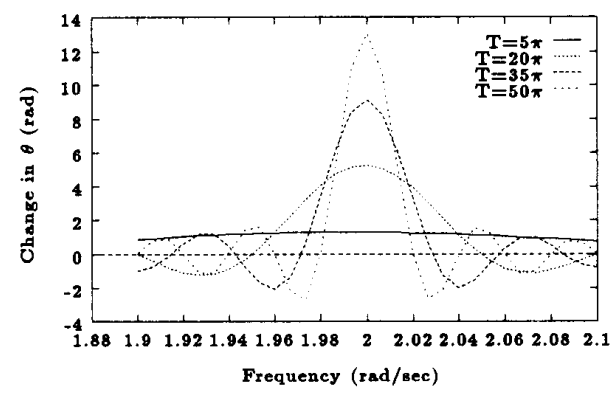

Figure 9: Magnitude of rotation vs $\omega_{\psi}$ for the $(2,1,1)$ gait

The "parking" gait: $(3,2,2)$

The final gait that was studied is that resulting from the frequency ratios $(3,2,2)$. In this gait a net displacement is produced in the $y$-direction. The position of the centre of mass is shown in Figure 10.

The following parameters were fixed for the analysis of the $(3,2,2)$ gait.

$$
\begin{aligned}
& a_{b},-a_{f}: 1 \mathrm{rad} \\
& \omega_{b}, \omega_{f}: 2 \mathrm{rad} / \mathrm{sec} \\
& \beta_{b}, \beta_{f}: 0 \mathrm{rad} \\
& a_{\psi}: 1 \mathrm{rad} \\
& \omega_{\psi}: 3 \mathrm{rad} / \mathrm{sec} \text { (unless otherwise specified) } \\
& \beta_{\psi}: 0 \mathrm{rad}
\end{aligned}
$$

The relationships between distance traveled in the $y-$ direction versus the phase angle, $\beta_{\psi}$, and the amplitude, $a_{\psi}$, are much the same as in the $(1,1,1)$ gait. The distance traveled in the $y$-direction versus the frequency is shown in Figure 11 .

Of course, there is further analysis yet to be performed on these gaits. Possible avenues of investigation will certainly include looking at cases where the wheels are driven out of phase, particularly $90^{\circ}$ out of phase (which has been seen as a working gait used in the actual Snakeboard); where only one of the wheels is driven (i.e. $\phi_{f}=0$ is fixed); and where the amplitude of the wheel oscillations is decreased as forward velocities increase (to reduce the oscillations seen in Figure 4). Future research may also include more extensive parameter sweeps than those described in this section, as well as the possible use of a variable inertia rotor (which would correspond to the rider's ability to move his arm's in and out). 


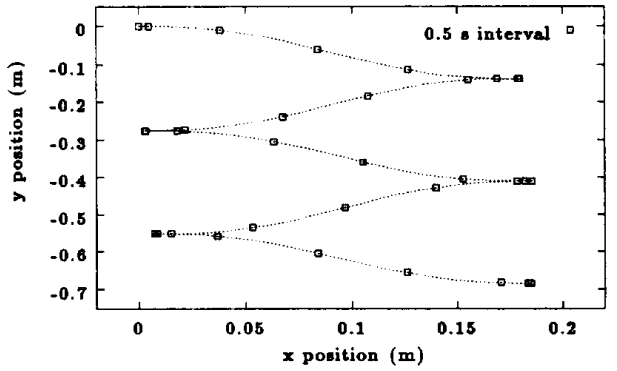

Figure 10: Position of the centre of mass for the $(3,2,2)$ gait

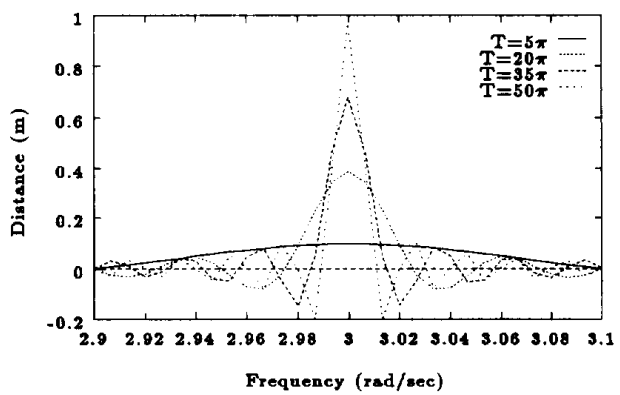

Figure 11: Distance traveled in the $y$-direction vs $a_{\psi}$ for the $(3,2,2)$ gait

\section{Discussion}

The Snakeboard example presented in this paper points to a number of future directions of research in the areas of locomotion and nonholonomic mechanics. While the Snakeboard shares a number of properties with other mechanical systems, the unique way in which motion is generated in this particular example has raised many new questions.

In some cases there is a natural principal bundle structure present, in which the geometry of the system can be studied by considering the lifting of paths in a certain base space into the total space. Roughly speaking, for these systems one can write the constraints as

$$
\dot{s}_{i}=\sum_{j=1}^{n-k} a_{i j}(r) \dot{r}_{j} \quad i=1, \ldots, k,
$$

where $q=(r, s) \in \mathbf{R}^{n-k} \times \mathbf{R}^{k}$ represents a splitting of the coordinates into base variables, $r$, and fibre variables, $s$. For such systems, one can generate paths for the system by specifying an initial condition and a path $r(t)$. The complete trajectory of the system is then determined by lifting the base path via equation (5.1) to a path in the fibre variables. For engineering systems, the base space is usually the space of controlled variables and hence $r(t)$ can be arbitrarily specified. For example, in a mobile robot one can take the base space to be the angular variables for the wheels. The fibre variables are the remaining configuration variables in the system and the velocity of the path in the base space gives a unique velocity for the fibre variables via the constraints.
For the snakeboard, a first-order lifting property is not possible. To see why this is so, suppose that a set of independent constraints of the form

$$
\dot{s}-A(r) \dot{r}=0
$$

where

$$
\begin{aligned}
& r=\left(\psi, \phi_{b}, \phi_{f}\right) \\
& s=(x, y, \theta) \\
& A(r) \in \mathbf{R}^{3 \times 3}
\end{aligned}
$$

were present. Then, if we begin with the base space variables at the origin and traverse a path which returns the base space variables to the origin and keeps them there (i.e., so that $\dot{r}=0$ after some time $T$ ), it follows that the time-derivatives of the fibre variables must be zero (i.e., $\dot{s}=0$ after the same time $T)$. However, this is not the case since it is possible to get the snakeboard moving in the forward direction while returning all control variables to the origin. Thus no such constraint can occur.

Note, however, that we can alternatively split the configuration variables into $(r, s)$, where $r=\left(\theta, \psi, \phi_{b}, \phi_{f}\right)$ and $s=(x, y)$. With this splitting the system fits into the form of (5.1). This formulation fails due to the fact that we cannot follow arbitrary paths in the base variables $\left(\theta, \psi, \phi_{b}, \phi_{f}\right)$, and have control of only three of the four control directions.

Current research by Bloch, et al. (see [3] and references therein), suggests a new framework for analysing this problem. The tools described in that paper utilise variational principles in order to blend the group invariance found in the unconstrained fibre bundle case with constraints which are nonholonomic. By using these tools, it is possible to describe motion along the fibre as being driven by a "pseudomomentum" term. This would differ from the traditional concept of momentum in that the "pseudo-momentum" would not be conserved, but instead would vary from point to point via a differential equation.

For the snakeboard, we can be somewhat more explicit, though this work is currently in progress and as such the exact formulation has not been settled upon. What we have found, however, is that by splitting the variables as above, with $s=(x, y, \theta)$ and $r=\left(\psi, \phi_{b}, \phi_{f}\right)$, we can arrive at an alternative expression relating the base and fibre variables. As mentioned above, the direct relationship expressed in (5.2) is not possible, but instead we can employ the momentum expression as a coupling term.

Let $p$ denote the constrained momentum, defined to be the momentum along trajectories which lie in the constraint distribution and are tangent to the group orbits (which are parameterised by $(x, y, \theta))$. Then, assuming the base variables to be the controlled variables, the differential equations governing the fibre and momentum terms take the form

$$
\begin{aligned}
& \dot{s}=g \xi(r, \dot{r}, p) \\
& \dot{p}=h(r) p+f(r) \dot{r},
\end{aligned}
$$

where $g$ represents the lifted group action of $S E(2)$ acting on velocities along the fibre. Notice that the fibre dependence of these equations occurs only in this lifted action. Thus, the solution to the momentum equation requires knowledge only of the path in the base space and the initial momentum. The momentum in turn provides the coupling which drives the motion along the fiber. Thus, in the case of the snakeboard, the process of lifting to the fiber is a two step process: first, the momentum is determined from the motion in the controlled variables, and second, the motion along the fiber 
is found using this information. Present work on the snakeboard has sought to further refine these relationships along with those developed in [3], and has provided valuable insight into the general problem of nonholonomic constraints in the presence of group symmetries.

An additional direction for future investigations is to examine the use of tools from geometric mechanics for studying other locomotion systems. A related piece of work is that of Shapere and Wilczek, who studied the locomotion of amoeba in highly viscous fluids [15]. They showed that it was possible to describe this motion using ideas from geometric mechanics and gauge theory. Following their lead, we have begun to investigate the possible use of geometric mechanics, and in particular the role of connections, in understanding other locomotion problems.

Finally, in terms of the snakeboard itself, there are a number of interesting questions related to motion planning. For example, one would like to be able to generate a set of inputs which moved the snakeboard from its starting configuration to some other given configuration. Many of the methods which have been developed for doing this for nonholonomic systems do not apply here since the system cannot be described solely in terms of a set of (Pfaffian) velocity constraints. Another direction for study is the optimal generation of inputs given finite energy considerations, similar to the work performed by Wilczek and Shapere (see [14]).

\section{Summary}

In this paper we have analysed gait patterns for a simplified model of the Snakeboard. We have found that while these gait patterns resemble those seen in many locomotion problems, previously used analysis techniques are not applicable to our model. The reason for the failure of the available tools is that the nonholonomic constraints couple with the natural inputs differently than is often seen. For this reason we feel that our model of the Snakeboard is representative of an interesting and important class of systems both in nonholonomic mechanics and in locomotion.

\section{References}

[1] M. Berkemeier and R. Fearing. Control of a two-link robot to achieve sliding and hopping gaits. In Proc. IEEE Int. Conf. on Robotics and Automation, pages 286-291, Nice, France, May 1992.

[2] Anthony M. Bloch and Peter E. Crouch. Kinematics and dynamics of nonholonomic control systems on Riemannian manifolds. In Proceedings of the 31st IEEE Confer. ence on Decision and Control, pages 1-5, Tucson, AZ, $\operatorname{dec} 1992$.

[3] Anthony M. Bloch, P. S. Krishnaprasad, Jerrold E. Marsden, and Richard M. Murray. Nonholonomic mechanical systems and symmetry, 1994.

[4] G.S. Chirikjian and J.W. Burdick. Kinematics of hyperredundant locomotion with applications to grasping. In Proc. IEEE Int. Conf. on Robotics and Automation, Sacramento, CA, April 1991. IEEE.

[5] Robert Hermann and Arthur J. Krenner. Nonlinear controllability and observability. IEEE Tansactions on $\mathrm{Au}$ tomatic Control, 22(5):728-740, 1977.
[6] S. Hirose and Y. Umetani. Kinematic control of active cord mechanism with tactile sensors. In Proc. $2^{\text {nd }}$ Int. CISM-IFT Symp. on Theory and Practice of Robots and Manipulators, pages 241-252, 1976.

[7] S. Kajita and K. Tani. Study of dynamic biped locomotion on rugged terrain. In Proc. IEEE Int. Conf. Robotics and Automation, pages 1405-1411, Sacramento, CA, April 1991. IEEE.

[8] D. Koditschek and M. Bühler. Analysis of a simplified hopping robot. Int. J. Robotics Research, 10(6):587-605, 1991.

[9] T. McGeer. Passive dynamic walking. Int. J. of Robotics Research, 9(2):62-82, Apr 1990.

[10] R.T. M'Closkey and J.W. Burdick. The periodic motions of a hopping robot with vertical and forward motion. Int. J. of Robotics Research, 12(3):197-218, Jun 1993.

[11] R. M. Murray and S. S. Sastry. Nonholonomic motion planning: Steering using sinusoids. IEEE Transactions on Automatic Control, 38(5):700-716, 1993.

[12] L. A. Pars. A Treatise on Analytical Dynamics. John Wiley and Sons, New York, New York, 1965.

[13] M.H. Raibert. Legged Robots that Balance. MIT Press, Cambridge, MA, 1986.

[14] Alfred Shapere and Frank Wilczek. Efficienies of selfpopulsion at low Reynolds number. J. Fluid Mech. 198:587-599, 1989.

[15] Alfred Shapere and Frank Wilczek. Geometry of selfpropulsion at low Reynolds number. J. Fluid Mech., 198:557-585, 1989.

[16] S. Song and K. Waldron. Machines that Walk: the Adaptive Suspension Vehicle. MIT Press, Cambridge, MA, 1989.

[17] D. Tilbury, R. M. Murray, and S. S. Sastry. Trajectory generation for the N-trailer problem using Goursat normal form. In Proceedings of the 32nd IEEE Conference on Decision and Control, pages 971-977, Dec. 1993.

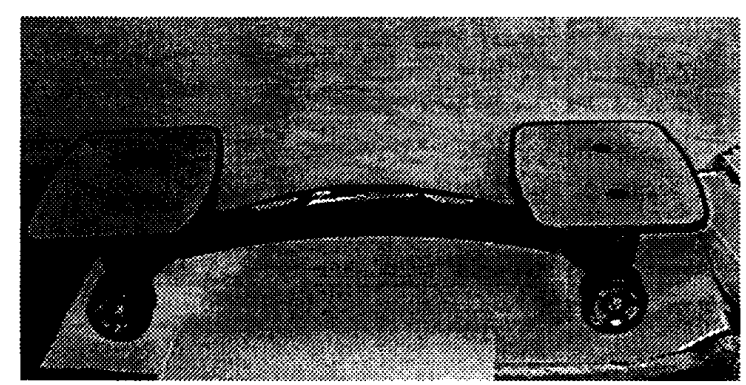

The Snakeboard 